\title{
Advances in Tooth Regeneration Techniques
}

Yoshikazu Mikami*

Department of Anatomy, School of Dentistry, Nihon University, Tokyo, Japan

\section{Introduction}

Periodontal disease, caries, and tooth fracture are the three main causes for tooth loss. Following tooth loss, artificial teeth have traditionally been used to restore masticatory function. Artificial tooth replacement methods such as implant treatment have been developed in recent years, but tooth regeneration, in which a new tooth is produced from cells, would be the ideal treatment. However, although 10 years have passed since Young et al. [1] first demonstrated in 2002 that teeth could be regenerated from cells, the technique is still in the animal trial stage. What is a problem at tooth regeneration? Is it possible to regenerate teeth from cells? In order to answer these questions, here, I present the latest advances in tooth regeneration.

\section{Dental Regenerative Medicine}

Regenerative medicine has always played an important role in the field of dentistry. In endodontics, for several decades, dentine regeneration has been induced by application of a calcium hydroxide preparation over inflamed pulp tissue in carious teeth. Guided tissue regeneration (GTR) was developed recently as a novel periodontal therapy, allowing regeneration of periodontal tissue without the use of cells or growth factors through the creation of space. While operative procedures have thus been established for partial tissue regeneration, research into regeneration of the whole tooth is still in the initial stage.

\section{Initial Research into Tooth Regeneration}

During development, enamel is formed from ectodermal epithelial tissue and dentine and pulp from mesenchymal tissue originating in the neural crest. Consequently, different types of cells are required to regenerate teeth. How, then, should we approach teeth regeneration techniques?

Honda et al. [2] attempted to regenerate teeth from cells taken from the mandibular third molar of 6-month-old pigs during the stage when the crowns form in the jawbone. They considered that this would simulate the physiological process as epithelial cells, pulp cells, and dental follicle cells are present in porcine teeth at this stage, and also because the developmental stage of porcine teeth at this time point matches the developmental stage of human third molars in childhood. They extracted porcine third molar tooth germs and harvested epithelial cells, pulp cells, and dental follicle cells, seeded the isolated cell groups onto a scaffold and transplanted them into the greater omentum of nude rats. When the transplants were taken out 20-30 weeks post-transplant, there had been regeneration of the tooth tissues such as enamel, dentine, and pulp. However, there was no regeneration of hard tissue when only tooth epithelial tissue cells were transplanted, and there was regeneration of dentine and bone but not of enamel when only tooth mesenchymal cells were transplanted. This shows that epithelium-mesenchyme interaction is required for tooth regeneration. Moreover, when the regeneration process was observed chronologically, immunostaining for the epithelial cell marker cytokeratin 14 at 6 weeks post-transplant revealed that epithelial cells showing a positive reaction to cytokeratin 14 were clumped together. However, at this point, the epithelial cells were negative for amelogenin antibody, an ameloblast marker. In other words, it appears that these epithelial cells were still undifferentiated, with no differentiation into ameloblasts. This indicates the existence of undifferentiated cells with the capacity for tooth regeneration in teeth during the period of crown formation.

This experiment was further repeated and the following problems were encountered: first, the tooth regeneration rate within the scaffold is low at just 50\%; second, multiple teeth are regenerated in a single scaffold; and last, while there is a small probability that the regenerated tooth will exhibit the same shape as a natural tooth, in almost all cases the regenerated tooth attains a shape different from that of a natural tooth. It will not be possible to clinical apply the above technique unless these problems are resolved. However, regeneration of teeth with shapes resembling those of natural teeth may occur, albeit with a low probability; it should therefore be possible to establish tooth regeneration techniques if undifferentiated cells with shape control function could be isolated from tooth germ.

\section{Tooth Regenerative Ability of Post-birth Tooth Germ Cells}

A possible cause for multiple tooth regeneration on a single scaffold is the method used for seeding the cells. In the method used by Honda et al. [2], isolated tooth germ epithelial cells and tooth germ mesenchymal cells were mixed and seeded on the scaffold; however, it is likely that the epithelium-mesenchyme interaction was induced irregularly at various locations within the scaffold, with the result that several teeth were regenerated. In their next experiment, Honda et al. [2] first seeded tooth pulp cells and then seeded tooth germ epithelial cells such that they were in contact with the tooth germ mesenchymal cells; these were then transplanted into the greater omentum of nude rats. Observation at 20 weeks post-transplant showed that there was one tooth in the scaffold, and the tooth tissue consisted mainly of dentine. Also, dentine regeneration was found to occur at an earlier stage than previously. Nonetheless, it was still difficult to regenerate teeth with the same shape as natural teeth.

\section{Recent Developments in Tooth Induction Methods}

Not just teeth but almost all organs develop from organ germs that are induced from epithelial and mesenchymal stem cells during development of the embryo. Organ germ is controlled by the epithelium-mesenchyme interaction. There have been attempts to develop a method of recreating the epithelium-mesenchyme

*Corresponding author: Yoshikazu Mikami, Department of Anatomy, School of Dentistry, Nihon University, 1-8-13 Kanda Surugadai Chiyoda-ku, Tokyo 101-8310, Japan, E-mail: mikami-t@dent.nihon-u.ac.jp

Received March 01, 2012; Accepted March 03, 2012; Published March 04, 2012

Citation: Mikami Y (2012) Advances in Tooth Regeneration Techniques. Dentistry 2:e105. doi:10.4172/2161-1122.1000e105

Copyright: (c) 2012 Mikami Y. This is an open-access article distributed under the terms of the Creative Commons Attribution License, which permits unrestricted use, distribution, and reproduction in any medium, provided the original author and source are credited. 
interaction in line with the program for organogenesis. In an experiment using mice, Nakao et al. [3] developed a three-dimensional cell manipulation technique that reorganizes embryonic ally-derived epithelial and mesenchymal stem cells by increasing the cell density and compartmentalizing the cells. Ikeda et al. [4] and Oshima et al. [5] transplanted the tooth germ regenerated by this technique into the molar extraction socket of adult mice. As the results, the regenerated tooth erupted and developed within the mouth with the same hardness as natural teeth and attachment with alveolar bone. Moreover, it was shown that these regenerated teeth were functional teeth that could feel pain as a result of external stimuli.

However, they made use of cells derived from embryos, so that if this method has to be clinically applied, a cell source that can be used in humans will have to be found. Embryonic stem (ES) cells and induced pluripotent stem (iPS) cells are very potential candidates. In particular, attention is focused on iPS cells, which are produced by introducing known factors into the body's somatic cells, as they can solve the ethical issues associated with ES cells and the problems of immune rejection associated with allogenic transplantation. However, preparation of iPS involves gene transfer, so there still remains the problem of canceration. At the same time, a number of stem cells have recently been isolated from wisdom teeth and deciduous teeth extracted for dental treatment, and they have attracted great interest in the field of regenerative medicine due to their high capacity for proliferation and differentiation. These cells may perhaps be a plausible cell source for tooth regeneration. In fact, Nakahara [6], recently reported a case of successful tooth regeneration using stem cells derived from extracted teeth by a method different from the aforementioned methods. Therefore, I next introduce the stem cells derived from extracted teeth that have been reported to date and outline the report by Nakahara [6].

Future of Regenerative Medicine Seen in Stem Cells from Extracted Teeth

Stem cells derived from teeth that are known to date are mainly of five types: dental pulp stem cells (DPSC), stem cells from exfoliated deciduous teeth (SHED), periodontal ligament stem cells (PDLSC), stem cells from apical papilla (SCAP), and dental follicle precursor cells (DFPC). SHED are isolated from the pulp of exfoliated deciduous teeth, while the other stem cells are derived from wisdom teeth. DPSC and PDLSC can be isolated from wisdom teeth obtained through routine extraction. Impacted wisdom teeth with incomplete root formation are occasionally extracted in young people; SCAP can be isolated from the apical pulp of such teeth, while DFPC can be isolated from the soft tissue surrounding the incomplete tooth. These stem cells derived from teeth have high capacity for proliferation and multipotent differentiation, and as there have recently been occasional reports of differentiation into neurons or muscle cells, it has become clear that they are similar to bone marrow stem cells.

Nakahara [6] successfully regenerated not only the tooth root but also periodontal membrane and alveolar bone, which are tooth supporting tissues, by wrapping mouse tooth crowns in a cell sheet prepared by culturing PDLSC obtained from extracted teeth. It had not previously been possible to induce tooth regeneration unless the artificial tooth germ produced by gathering tooth germ cells was transplanted into animals. Previous tooth regeneration methods used embryo cells and would therefore be problematic from the point of view of clinical application. However, the study by Nakahara [6] opens up the possibility of forming tooth root and periodontal tissue through ex vivo culture of materials that can be readily acquired in clinical practice. Further developments are expected in the future.

\section{Conclusion}

Teeth in the developmental stage were shown to have the ability to regenerate a tooth once again, and this discovery marked the start of tooth regeneration research. Recently, it was further discovered that a new tooth can be regenerated from an extracted tooth, but there still remain many issues to be solved before its clinical application. Nonetheless, technology to regenerate teeth from cells will probably be established in the near future, because the issues which should be solved are becoming clear.

\section{Acknowledgments}

I am grateful to Dr. Honda MJ and Dr. Omoteyama K Nihon University, School of Dentistry, Department of Anatomy, for their encouragement.

\section{References}

1. Young CS, Terada S, Vacanti JP, Honda M, Bartlett JD, et al. (2002) Tissue engineering of complex tooth structures on biodegradable polymer scaffolds. $J$ Dent Res 81: 695-700.

2. Honda MJ, Sumita $Y$, Kagami H, Ueda M (2005) Histological and immunohistochemical studies of tissue engineered odontogenesis. Arch Histol Cytol 68: 89-101.

3. Nakao K, Morita R, Saji Y, Ishida K, Tomita Y, et al. (2007) The development of a bioengineered organ germ method. Nat Methods 4: 227-230.

4. Ikeda E, Morita R, Nakao K, Ishida K, Nakamura T, et al. (2009) Fully functiona bioengineered tooth replacement as an organ replacement therapy. Proc Nat Acad Sci U S A 106: 13475-13480.

5. Oshima M, Mizuno M, Imamura A, Ogawa M, Yasukawa M, et al. (2011) Functional tooth regeneration using a bioengineered tooth unit as a mature organ replacement regenerative therapy. PLoS One 6: e21531.

6. Nakahara T (2009) Shourani no shikairyou ha dounarunoka? J Tokyo Dent Assoc 57: 3-10. 